\title{
A Novel Chemical Compound for Inhibition of SARS Coronavirus Helicase
}

\author{
Jin-Moo Lee ${ }^{1 \dagger}$, Jin-Beom Cho ${ }^{1 \dagger}$, Hee-Chul Ahn ${ }^{2}$, Woong Jung ${ }^{3}$, and Yong-Joo Jeong ${ }^{1 *}$ \\ ${ }^{1}$ Department of Applied Chemistry, Kookmin University, Seoul 02707, Republic of Korea \\ ${ }^{2}$ Department of Pharmacy, Dongguk University-Seoul, Gyeonggi-do 10326, Republic of Korea \\ ${ }^{3}$ Department of Emergency Medicine, Kyung Hee University Hospital at Gangdong, Seoul 05278, Republic of Korea
}

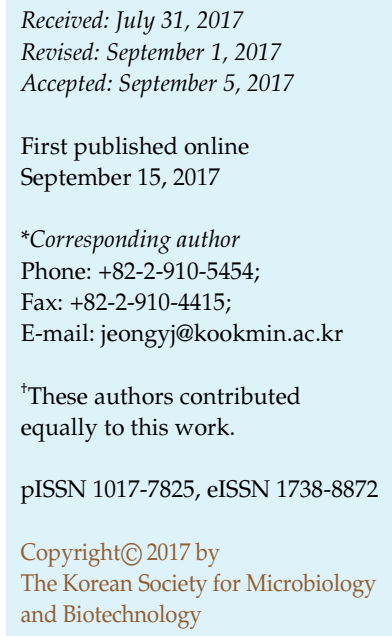

We have discovered a novel chemical compound, (E)-3-(furan-2-yl)- $N$-(4-sulfamoylphenyl) acrylamide, that suppresses the enzymatic activities of SARS coronavirus helicase. To determine the inhibitory effect, ATP hydrolysis and double-stranded DNA unwinding assays were performed in the presence of various concentrations of the compound. Through these assays, we obtained $\mathrm{IC}_{50}$ values of $2.09 \pm 0.30 \mu \mathrm{M}$ (ATP hydrolysis) and $13.2 \pm 0.9 \mu \mathrm{M}$ (DNA unwinding), respectively. Moreover, we found that the compound did not have any significant cytotoxicity when $40 \mu \mathrm{M}$ of it was used. Our results showed that the compound might be useful to be developed as an inhibitor against SARS coronavirus.

Keywords: SARS, helicase, ATP hydrolysis, dsDNA unwinding, cytotoxicity
In 2002, severe acute respiratory syndrome (SARS) broke out in Guangdong Province of China and spread rapidly to many regions. According to the World Health Organization, almost 800 victims were reported and the fatality rate of SARS was about $10 \%$. Fortunately, SARS was controlled in a year and further reoccurrence has not been reported. However, SARS is still regarded as a great concern to human health because effective medicines or vaccinations have not been developed. Therefore, the efforts to develop successful anti-SARS agents should be continued for possible reappearance of threatening SARS.

In general, coronaviruses are enveloped and their genome is single-stranded positive-sense RNA. They were not regarded as fatal before SARS appeared. In fact, a few human coronaviruses, such as HCoV-229E along with OC43, have been known as causative agent of the common cold. However, SARS was caused by an unprecedented coronavirus, SARS coronavirus (SCV), with a genome of about $29.7 \mathrm{~kb}[1,2]$. Of the many genes of SCV, the $21.2 \mathrm{~kb}$ replicase gene, which is located at the 5 '-end of the SCV genome, is responsible for producing two polyproteins, pp1ab and pp1a [3, 4]. The two polyproteins are subsequently cleaved by viral proteinases and produce several nonstructural proteins (nsPs) [3, 5]. It is noted that NTPase/helicase (nsP13), the target protein in this study, is indispensable for SCV to replicate and a member of the membrane-bound viral replicase complex $[5,6]$. In fact, viral polymerase and helicase have been regarded as potential target proteins to identify new antiviral agents [7-9]. Helicases are molecular motors that translocate along single-stranded (ss) nucleic acid or unwind double-stranded (ds) nucleic acid using the energy released from nucleoside triphosphate (NTP) [10, 11]. Owing to the importance of helicase in genome replication, most organisms, from bacteria to human beings, encode helicases [12]. Many viruses also have their own helicases. Although the function of helicases seems to be similar in almost all organisms, the working mechanism is very unique from species to species. In terms of SCV helicase, we have acquired a lot of mechanistic characteristics during the past years [13-15]. Based on the accumulated knowledge, we have kept going to find inhibitory materials against SCV helicase, such as small molecules [13, 16, 17]. 


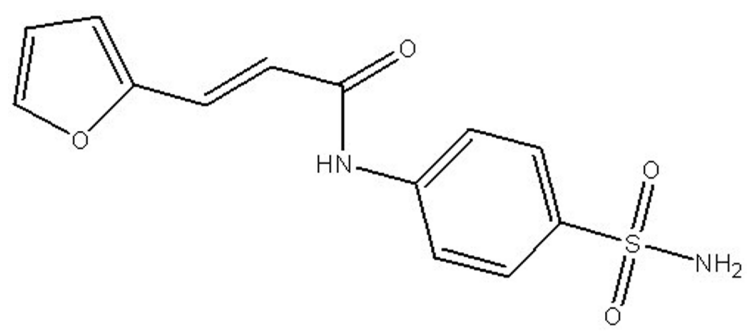

Fig. 1. Chemical structure of (E)-3-(furan-2-yl)-N-(4sulfamoylphenyl)acrylamide.

In the present study, we have identified a novel small molecule from an in-house library, designated as FSPA hereafter, with the structure (E)-3-(furan-2-yl)-N-(4sulfamoylphenyl)acrylamide (Fig. 1), that can suppress both ATP hydrolysis and dsDNA unwinding activities of SCV helicase. Moreover, FSPA does not have significant cytotoxic effect on human WI-38 cells.

The SCV helicase expression vector was transformed in E. coli and overexpressed, and the protein was purified as described previously [18]. We measured the ATP hydrolysis activity of the purified helicase by monitoring the released inorganic phosphate [19]. Based on the fact that SCV helicase hydrolyzes the ATP substrate during the translocation on ssDNA M13, we were able to screen the chemical compounds that inhibit the function of ATP hydrolysis. A $25 \mu \mathrm{l}$ solution containing $400 \mathrm{nM}$ SCV helicase and $0.5 \mu \mathrm{l}$ of various concentrations of FSPA in $50 \mathrm{mM}$ Tris- $\mathrm{HCl}(\mathrm{pH}$ 6.6) buffer was mixed with an equal volume of solution containing $100 \mathrm{mM} \mathrm{NaCl}, 10 \mathrm{mM} \mathrm{MgCl}, 4 \mathrm{mM} \mathrm{ATP}$, and $4 \mathrm{nM}$ circular ssDNA M13 in $50 \mathrm{mM}$ Tris- $\mathrm{HCl}(\mathrm{pH}$ 6.6) buffer in the well of a 96-well plate. The total $50 \mu$ solution was incubated for $10 \mathrm{~min}$ at $37^{\circ} \mathrm{C}$ and the reaction was stopped by adding $200 \mu \mathrm{l}$ of $\mathrm{AM} / \mathrm{MG}$ reagent $(0.034 \%$ malachite green, $1.05 \%$ ammonium molybdate, and $0.04 \%$ Tween 20 in $1 \mathrm{M} \mathrm{HCl})$. The released inorganic phosphate complexed with $\mathrm{AM} / \mathrm{MG}$ reagent developed a deep blue color, which was measured at $620 \mathrm{~nm}$. The accurate amount of inorganic phosphate was calculated by a standard curve. Fig. 2A shows the inhibition percentage of ATP hydrolysis in the presence of various concentrations of FSPA. After fitting the curve, $\mathrm{IC}_{50}$ value of FSPA was determined to be $2.09 \pm 0.30 \mu \mathrm{M}$. Further experiments were carried out to investigate the dsDNA unwinding by SCV helicase in the presence of FSPA. DNA unwinding experiment was designed on the basis of fluorescence resonance energy transfer (FRET). Two different fluorescent dyes, fluorescein (FAM) and carboxytetramethylrhodamine (TAMRA), are labeled to each strand of the dsDNA. When the two DNA strands are annealed by base pairing, no fluorescence is detected because a constant FRET occurrs between FAM and TAMRA. However, this FRET is lost when the annealed dsDNA is unwound by helicase. Using this experimental approach, dsDNA unwinding was measured in the presence of FSPA. Briefly, the 3'-terminus TAMRAmodified oligomer (45-base-oligomer) was annealed to the 5'-terminus FAM-modified oligomer (25-base-oligomer), which produced a partial annealed dsDNA substrate containing 25 base pairs and a single-stranded $20 \mathrm{dT}$ overhang at the 5 '-terminus as described previously [19]. A $80 \mu \mathrm{l}$ solution containing $150 \mathrm{nM}$ SCV helicase and $1 \mu \mathrm{l}$ of various concentrations of FSPA in 20 mM HEPES ( $\mathrm{pH} 7.4$ ) was mixed with a $20 \mu \mathrm{l}$ solution containing $5 \mathrm{mM} \mathrm{MgCl}_{2}$, $45 \mathrm{mM}$ ATP, $25 \mathrm{mM}$ DTT, and $100 \mathrm{nM}$ dsDNA substrate in $20 \mathrm{mM}$ HEPES ( $\mathrm{pH}$ 7.4) buffer in the well of a 96-well plate. After 2 min incubation at $37^{\circ} \mathrm{C}$, the dsDNA unwinding reaction was terminated by adding a $100 \mu \mathrm{l}$ solution containing 0.1 M EDTA and $0.4 \mu \mathrm{M}$ trap DNA (unmodified
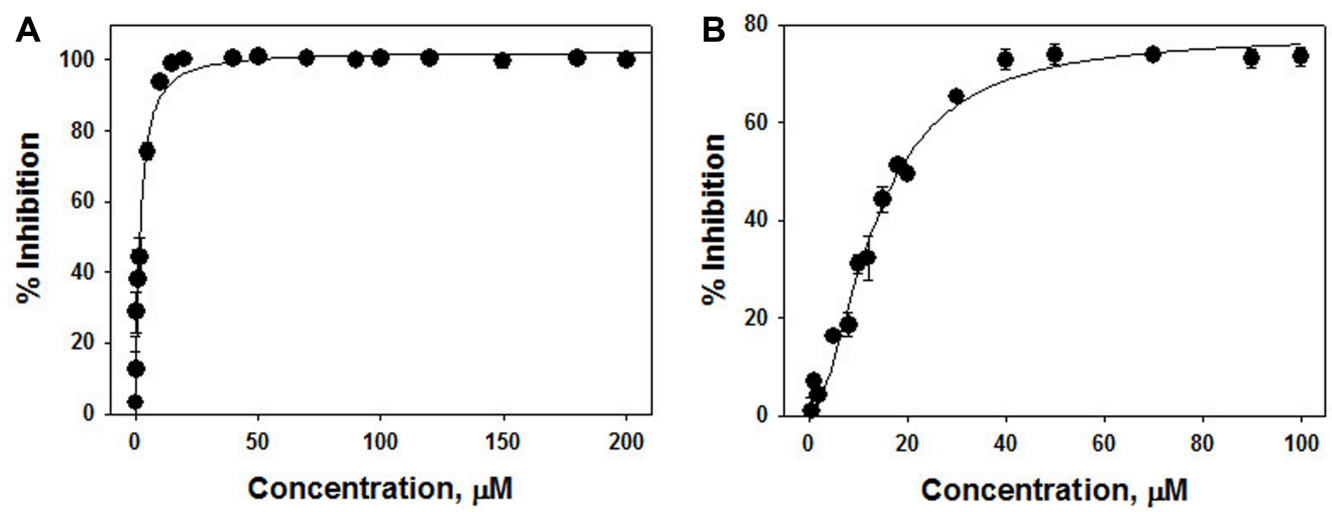

Fig. 2. Percentage inhibition of SCV helicase by (E)-3-(furan-2-yl)-N-(4-sulfamoylphenyl)acrylamide.

(A) ATP hydrolysis activity. (B) dsDNA unwinding activity. 


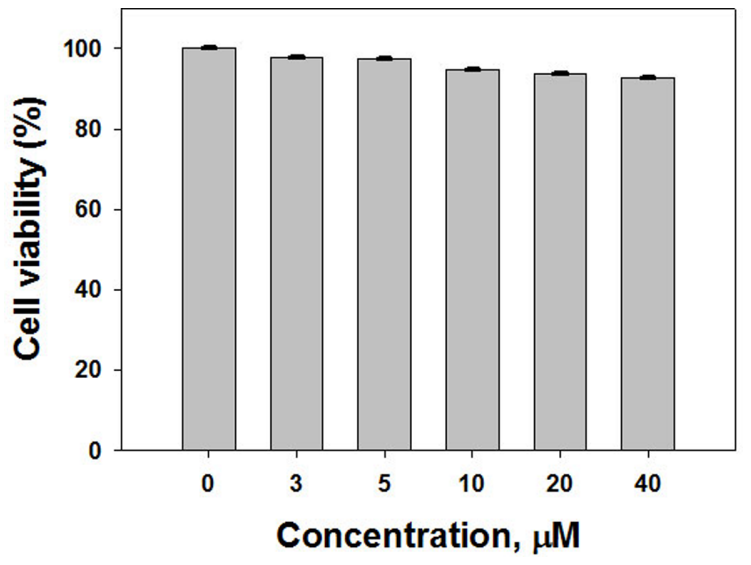

Fig. 3. Measurement of cytotoxicity in the presence of (E)-3(furan-2-yl)-N-(4-sulfamoylphenyl)acrylamide.

25 bases oligomer) in $20 \mathrm{mM}$ HEPES (pH 7.4). The trap DNA, which was added excessively compared with the amount of dsDNA substrate, was used to prevent reannealing between the two fluorescent dye-labeled DNA strands. The fluorescence signal was monitored by measuring at $535 \mathrm{~nm}$ after the mixture was excited at $485 \mathrm{~nm}$. Fig. 2B shows the inhibition percentage of dsDNA unwinding in the presence of various concentrations of FSPA. After fitting the curve, the $\mathrm{IC}_{50}$ value of FSPA was determined to be $13.2 \pm 0.9 \mu \mathrm{M}$. Finally, we examined the cytotoxicity of FSPA using WI-38 cells (human fetal lung cells). The cells were maintained at $37^{\circ} \mathrm{C}$ in an incubator with atmosphere of $5 \% \mathrm{CO}_{2}$ and cultured in DMEM containing 10\% heat-inactivated fetal calf serum, $1 \%$ non-essential amino acid, streptomycin $(100 \mu \mathrm{g} / \mathrm{ml})$, and penicillin $(100$ units $/ \mathrm{ml})$. After $24 \mathrm{~h}$ incubation with starting $6 \times 10^{4}$ cells $/ \mathrm{ml}$ density in a 96well plate, final $3-40 \mu \mathrm{M}$ concentrations of FSPA were added to the cells. We further incubated the cells for $48 \mathrm{~h}$, and then $50 \mu$ of 3-(4,5-dimethylthiazol-2-yl)-2,5-diphenyltetrazolium bromide (MTT) stock solution $(2 \mathrm{mg} / \mathrm{ml})$ was added to each well to make a total reaction volume of $250 \mu$ l. After $2 \mathrm{~h}$ incubation, the supernatant was discarded and the produced formazan crystal was dissolved in $150 \mu \mathrm{l}$ of dimethyl sulfoxide. The developed purple color was monitored by measuring the absorbance at $540 \mathrm{~nm}$ using a scanning multiwell spectrophotometer. As shown in Fig. 3, FSPA does not have significant cytotoxicity at any concentration tested.

In conclusion, we have identified FSPA as a novel chemical inhibitor in this study, as an ongoing effort to discover new small molecules that suppress the activity of SCV helicase. In fact, we have previously reported that aryl diketoacids and dihydroxychromone derivatives block only dsDNA unwinding, not ATP hydrolysis activity of SCV helicase $[13,16]$. On the other hand, in the cases of myricetin and scutellarein, as a result of natural compounds screening, only ATP hydrolysis was inhibited without affecting the dsDNA unwinding activity [17]. However, the reason for this discrepancy is not clear at present. Interestingly, FSPA, the topic of this study, was able to inhibit both activities. Due to the absence of threedimensional structure information about SCV helicase, as of yet, it is also hard to explain how FSPA elicits the inhibitory effect on the activity of SCV helicase. A more detailed molecular simulation analysis will be necessary to understand the inhibitory mechanism of each molecule. It is also noted that FSPA resulted in an over 93\% cell viability of the human fetal lung cell line up to the $40 \mu \mathrm{M}$ concentration used, indicating that the compound has no significant cytotoxicity. Collectively, we propose that FSPA has great promise for use as an anti-SARS agent, although future in vivo studies would be necessary.

\section{Acknowledgments}

This study was supported by the Basic Science Research Program through the National Research Foundation of Korea (NRF) funded by the Ministry of Education (NRF2017R1A2B4003251).

\section{References}

1. Marra MA, Jones SJ, Astell CR, Holt RA, Brooks-Wilson A, Butterfield YS, et al. 2003. The genome sequence of the SARS-associated coronavirus. Science 300: 1399-1404.

2. Rota PA, Oberste MS, Monroe SS, Nix WA, Campagnoli R, Icenogle JP, et al. 2003. Characterization of a novel coronavirus associated with severe acute respiratory syndrome. Science 300: 1394-1399.

3. Lai MM, Cavanagh D. 1997. The molecular biology of coronaviruses. Adv. Virus Res. 48: 1-100.

4. Ziebuhr J, Snijder EJ, Gorbalenya AE. 2000. Virus-encoded proteinases and proteolytic processing in the Nidovirales. J. Gen. Virol. 81: 853-879.

5. Ivanov KA, Thiel V, Dobbe JC, van der Meer Y, Snijder EJ, Ziebuhr J. 2004. Multiple enzymatic activities associated with severe acute respiratory syndrome coronavirus helicase. J. Virol. 78: 5619-5632.

6. Tanner JA, Watt RM, Chai YB, Lu LY, Lin MC, Peiris JS, et al. 2003. The severe acute respiratory syndrome (SARS) coronavirus NTPase/helicase belongs to a distinct class of $5^{\prime}$ to 3' viral helicases. J. Biol. Chem. 278: 39578-39582.

7. Anand K, Ziebuhr J, Wadhwani P, Mesters JR, Hilgenfeld R. 2003. Coronavirus main proteinase (3CLpro) structure: basis 
for design of anti-SARS drugs. Science 300: 1763-1767.

8. Borowski P, Schalinski S, Schmitz H. 2002. Nucleotide triphosphatase/helicase of hepatitis $\mathrm{C}$ virus as a target for antiviral therapy. Antiviral Res. 55: 397-412.

9. Holmes KV. 2003. SARS coronavirus: a new challenge for prevention and therapy. J. Clin. Invest. 111: 1605-1609.

10. Patel SS, Donmez I. 2006. Mechanisms of helicases. J. Biol. Chem. 281: 18265-18268.

11. Patel SS, Picha KM. 2000. Structure and function of hexameric helicases. Annu. Rev. Biochem. 69: 651-697.

12. Matson SW, Bean DW, George JW. 1994. DNA helicases: enzymes with essential roles in all aspects of DNA metabolism. Bioessays 16: 13-22.

13. Lee C, Lee JM, Lee NR, Jin BS, Jang KJ, Kim DE, et al. 2009. Aryl diketoacids (ADK) selectively inhibit duplex DNAunwinding activity of SARS coronavirus NTPase/helicase. Bioorg. Med. Chem. Lett. 19: 1636-1638.

14. Lee NR, Kwon HM, Park K, Oh S, Jeong YJ, Kim DE. 2010. Cooperative translocation enhances the unwinding of duplex DNA by SARS coronavirus helicase nsP13. Nucleic Acids Res. 38: 7626-7636.
15. Lee NR, Lee AR, Lee B, Kim D-E, Jeong YJ. 2009. ATP hydrolysis analysis of severe acute respiratory syndrome (SARS) coronavirus helicase. Bull. Korean Chem. Soc. 30: 1724-1728.

16. Lee C, Lee JM, Lee NR, Kim DE, Jeong YJ, Chong Y. 2009. Investigation of the pharmacophore space of severe acute respiratory syndrome coronavirus (SARS-CoV) NTPase/ helicase by dihydroxychromone derivatives. Bioorg. Med. Chem. Lett. 19: 4538-4541.

17. Yu MS, Lee J, Lee JM, Kim Y, Chin YW, Jee JG, et al. 2012. Identification of myricetin and scutellarein as novel chemical inhibitors of the SARS coronavirus helicase, nsP13. Bioorg. Med. Chem. Lett. 22: 4049-4054.

18. Jang KJ, Lee NR, Yeo WS, Jeong YJ, Kim DE. 2008. Isolation of inhibitory RNA aptamers against severe acute respiratory syndrome (SARS) coronavirus NTPase/helicase. Biochem. Biophys. Res. Commun. 366: 738-744.

19. Cho JB, Lee JM, Ahn HC, Jeong YJ. 2015. Identification of a novel small molecule inhibitor against SARS coronavirus helicase. J. Microbiol. Biotechnol. 25: 2007-2010. 